\title{
Neuroplastic Control of Developmental Stutter via the Frontal Aslant Tract: Past, Present, and Future
}

\author{
Hilary Mc Donagh*and Kenneth Monaghan \\ School of Science, Institute of Technology (IT) Sligo, Ireland
}

*Corresponding author: Hilary Mc Donagh, Clinical Health and Nutrition Centre (CHANCE), Neuroplasticity Research Group (NRG), School of Science, Institute of Technology (IT) Sligo, Ireland.

To Cite This Article: Hilary Mc Donagh. Neuroplastic Control of Developmental Stutter via the Frontal Aslant Tract: Past, Present, and Future. Am J Biomed Sci \& Res. 2019 - 5(6). AJBSR.MS.ID.000978. DOI: 10.34297/AJBSR.2019.05.000978.

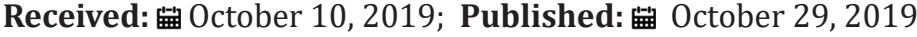

\author{
Abstract \\ An innovative new approach to the management of Developmental Stutter applying the principles underlying neuroplasticity to the unconscious \\ neural mechanics influencing tongue movement.
}

Keywords: Tongue; Neuroplasticity; Developmental Stutter; Frontal Aslant Tract; Saccades; Nucleus Basalis; Critical Period; Unconscious

\section{Introduction}

\section{Language}

There is a common perception that "Language" is "what makes us human" [1]. The ability to manipulate our tongue and vocal cords and produce a great variety of sounds had evolved in humans by 400,000 years ago [2]. Without this ability, vocalisations would be limited to "aah", "ooh" and "um" [3]. It is likely that in conjunction with producing a new range of sounds, language evolved [2]. Language has enabled humans to communicate real (e.g. laws) and imagined (e.g. belief systems) order, so changing the course of evolution for our species and our planet.

\section{Movement}

Verbal communication has been replaced to some extent with digital, electronic and written media, but face to face conversation remains the most powerful as the human facial expressions and hand gestures add intimacy, meaning and context to spoken language. It is actually these actions that shape our thoughts [4] and paying attention to these systems in our environments, make us better communicators Tversky [4] believes that movements transcend language as a basis for consciousness.

\section{Developmental Stutter}

Children acquire language initially through babbling repeating the sounds, pitch and rhythm of the language they have been exposed to. By age two children typically have a vocabulary of about 200 words [5]. While it is normal for most children to experience mild disruptions in the fluency of speech between the age of two and seven, for some children this continues into school years and even adulthood. This condition is called Developmental Stutter (DS). Characteristics of DS include repetitions of syllables, words or phrases, interjections, revisions, prolongations and tense pauses. The experience of DS can include avoidance of sounds, words, people and even places [6]. People who stutter say that they prefer not to talk at all rather than allowing others to hear that they stutter [7]. Developmental Stutter and its range of associated behaviours can result in reduced quality of life and well-being [8]. In childhood, the incidence of DS is about $5 \%$, and its recovery rate is up to about $80 \%$, resulting in a prevalence of persistent DS in about $1 \%$ of the adult population [9]. For individuals with DS the inability to speak the words they are thinking is therefore one of life's cruelest inflictions.

Past

Even though reports of humans stuttering date back to Demosthenes in $354 \mathrm{BC}$, it is only in the last decade that this silenced $1 \%$ of the world's population have been given a voice. This has been helped particularly by the Movie the "Kings Speech" which depicted living with a DS and the human trauma associated with the debilitating condition. Historically, theories concerning the cause of DS have ranged from dryness of tongue to abnormal speech apparatus, while in the 20th century DS was thought to be a psychogenic disorder [10]. Today, approaches used by Speech 
and Language professionals in the management of DS vary greatly based on training, experience, and theoretical/philosophical beliefs about its cause [11].

\section{Present}

For childhood DS, interventions are typically behavioural [12]. Existing approaches can be classified as either direct or indirect. The direct approach works directly with the child; to modify behaviour patterns when stuttering occurs, the indirect with caregivers and the speaking environment; to modify situational factors. Both are supported by clinical trials and a current international research project is attempting to establish which is most effective [13].

\section{Future}

There appears to be a need for new innovative treatments. One concept being currently investigated [14] concentrates on the neurological systems effecting tongue movement and how to control them. For speech to occur we learn how to consciously manipulate our tongue. These conscious, learned movement patterns are then stored and replayed on demand. However, our conscious is not the only part of our brain influencing tongue manipulation and positioning. In 2012, Catani and his colleagues [15] named a newly identified white matter cortical pathway, the Frontal Aslant Tract (FAT) within the brain. This is only recently visible thanks to advances in neuroimaging technology. While the existence of the FAT has been established in humans and non-human primates, in humans it is larger in the left hemisphere and includes Broca's area, long identified as a structure unique to human brains and necessary for language. The role of the FAT in DS was outlined by Dick. In the report, [16] identified three forces: limb movements, the ocular motor system and conscious control as competing for control of the motor system, via the FAT and highlighted a need for us to connect our mechanical functioning with our neurological functioning.

This new approach considers that if we have three systems trying to control our motor system (specifically our tongue), conscious movements will be enhanced if the three systems (forces) work together rather than competing. While we might not be consciously aware of it, our tongue moves all the time and only slows down to enable speech [17]. If we consider that all movement is in response to neurological signals, the obvious question is how do the unconscious systems (ocular/limb) influencing tongue movements operate? This research concept aims to examine the operation of these systems.

\section{Mechanical functioning: Tongue Movement Operating Systems}

\section{The first operating system - Limb Movements}

The first brain operating system influencing tongue movement is instinctive, present at birth. These movements are reflexive and related to body movement and survival. They originate from the oldest part of our brain: the brain stem. Many of our primary reflexes have been linked with tongue movement: rooting, suckling, tongue, swallow, bite, transverse tongue and gag [18]. Linking primitive tactile reflexes and reflexes of position with tongue movement is an area that does not appear to have been theoretically explored, probably because these reflexes are suppressed during the first few months of life and replaced with postural reflexes [19]. We consciously learn how to move our limbs in space before we learn to speak.

\section{Critical Period for Learning}

Within the brain, there is a critical period for language acquisition that occurs up to about age three [20]. In early childhood, our brain is its most plastic and the nucleus basalis, crucial for forming new associations [21], is always activated. By two years of age the basic neural architecture to support how to focus attention and achieve a goal, and how to reorient to unexpected but relevant stimuli in the environment, [22] is in place. Through practice, the neural connections for learned, repeated movement patterns become stronger and faster. The critical period for ocular dominance starts at a young age and closes around age seven [23]. This is commonly the period when developmental stuttering commences. The critical period for development of the FAT appears to be up to 6 years during which time micro structural changes occur quickly. The FAT does not change much after age 6 except for normal age-related changes from age 11 on [24].

\section{Second operating system - ocular motor}

The second operating system is our ocular motor system. Even though we are not consciously aware of it our eyes are constantly on the move. These movements are called saccades and are directly linked with our learned behaviour patterns / unconscious programming. As our unconscious considers options and prepares us for action, our eyes identify the targets and vectors of possible movements [25]. These lightning fast movements are called saccades. Saccades can be described as the quick simultaneous movements of both eyes between two fixation points. These eye movements last between $20-200$ milliseconds. As we spend 5\% of our day mindful of our current environment, our unconscious is constantly trying to predict what action we will do next and filling in blanks about what our environment is like. By searching through our "memory "database of automated /learned actions, our unconscious decides what is most likely to occur given the current stimuli and past experience. As our eyes are constantly on the move, our unconscious is also predicting what we are seeing, and our conscious experience of the world is a combination of reality and virtual reality [26]. Our perception of reality is a combination of the "real" and the "expected". As we are using our conscious brains to access memories and imagine scenarios, our unconscious is filling in the gaps based on our experience. This idea of predictive coding assumes that perception is an iterative process between prior knowledge and sensory feedback [27]. 


\section{Unconscious Eye Movement and Unconscious Tongue Movement}

Both our eyes [25] and our tongue [17] are constantly on the move without our conscious awareness. It may seem unclear how eye movements effect speech and tongue movement, but the mechanics behind this were outlined by [14]. If you flatten the back of your tongue on the roof of your mouth and move your eyes from side to side, you will feel the back of your tongue mirror your eye movements. This is because our brain is predicting that we might move in the direction of our gaze and preparing our body to do so. This effect also works in reverse, if you move your tongue from side to side while reading you will feel your eyes move slightly. This effect and indeed the effect of our tongue moving in response to our limbs moving can only be experienced when our tongue is touching the roof our mouth. When the tongue position is lower in the mouth the constant movement goes unnoticed.

\section{Third operating system - conscious movement and new learning}

The third system trying to control tongue movement is our conscious control or executive function. It is estimated that we spent approx. $5 \%$ of our time consciously aware of our movements. Speech involves replaying pre-stored motor programmes. For people with DS the pre-stored programmes do not produce clear speech. For the brain to focus on a new specific task, we must pay attention to it. To correct speech dysfluencies after the critical period when learning is automatic, to learn new skills, we must activate the nucleus basalis so we can strengthen new neural connection [21]. The nucleus basalis projects into Broca's area which is part of the FAT. After the critical period, behavioral and context outcomes regulate plasticity: focused attention activates the nucleus basalis [28], unexpected events lead to the release of Noradrenaline to amplify their novelty [29] and additionally, Dopamine is released when we predict a reward [30].

As all three systems are influencing tongue movement, our executive function must be able to override the other two systems to successfully manipulate tongue movement and produce smooth speech. Out of the three operating systems, limb movements, eye movements and conscious control, our eye movements occur first as they concern predictions. Consequently, logic would suggest that changing the direction of our gaze changes predictions. This is the fundamental basis of this current concept that changing eye movement would make logical sense as it is the precursor to DS.

\section{Saccadic Eye Movement Studies}

Saccadic eye movements are immediately altered by changing their starting position [31], thereby causing us to pay attention. Modification of saccadic eye movements has been directly linked to neuroplasticity [32]. It is important that we are aware of the predictive nature of DS as anticipation of DS is a core aspect of the stuttering experience [33]. In a reading task, stutterers' eyes fixate on the words on which they anticipate difficulty [34]. The anticipation of the stutter has already altered the tongues starting position long before the stutter occurs. The links between the three systems are evident as unusual eye movement and hand gestures typically accompany stuttering behaviour. Indeed, for an evolutionary neurological perspective, both language and tool use (involving limb movements) share parts of Broca's area [35]. If we do not alter the prediction of stuttering, the experience of stuttering becomes a self-fulfilling prophecy as the tongue has already moved making the production of clear speech difficult.

In a UK Randomised Control Trial (RCT) study by [36] of 69 adults (16male) aged (27.5 \pm 9.5 yrs.), 23 were assigned to three eye conditions, bilateral saccades, vertical saccades and no eye movement. Standardized testing showed bilateral saccades enhanced autobiographical memory fluency for retrieval of episodic information. In a., French study, ninety-five healthy children were assigned to 5 groups: Group $1 \mathrm{n}=19$ aged 5-7 years (mean age: 6.4460.08 years); Group $2 \mathrm{n}=22$ children aged $7-9$ years (mean age: 7.6260.13); Group $3 \mathrm{n}=1616$ children aged 9-11 years (mean age: 9.5860.12); Group $4 \mathrm{n}=20$ children aged $11-14$ years (mean age: 12.1960.16) and Group $5 \mathrm{n}=18$ children aged 14-18 years (mean age: 15.0460.20). All participants were tested on two visual tasks fixations and saccades and postural stability was measured using a platform. One of their findings showed that performing saccades improves postural stability with respect to a simple task (fixation) regardless of age. A review of the role of Eye Movement Desensitization Reprocessing Therapy in the treatment of both psychological and somatic disorder [37], 20 RCT indicated positive effects of bilateral eye movements in the desensitization stage of the therapy. Twelve randomized studies of the eye movement component noted rapid decreases in negative emotions and/or vividness of disturbing images, with an additional 8 reporting a variety of other memory effects.

Moving your eyes from side to side on a horizontal plane creates a mid-point or "average" tongue position, on the roof of the mouth. There is a growing body of research suggesting that this specific tongue position is optimal for body functioning. This evidence spans many disciplines: malocclusion and posture [38], heart function [39], muscle strength [40].

\section{New Approach to Developmental Stutter}

The link between this tongue position and our primary reflexes may be of great importance. As we habitually develop a dominant hand, eye, foot, and our body posture slowly move off centre, which effects functioning and development. This is because our skeletal muscles have a memory and do not return to their original starting position, but the average of our last movement. The long-term effects of this process can be clearly seen in the study of facial symmetry [41]. To nullify this incorrect learned predicted movement, it is necessary to use both eyes equally and 
be centred- this can be achieved by looking down in the direction of your navel. The movement required is only concerning your eyes, not your head. This redirection of gaze causes the posterior part of the tongue to be placed in the optimal position previously described. For DS, the downward gaze is required only for the start of the word. This novel approach explores modifying the unconscious neural programs responsible for tongue movement and positioning. From a chronological perspective, after the critical period, the first operating neurological system to activate and control our movements, in response to any stimulus, is the ocular motor system.

The viability of this novel approach has already been explored. In 2018, McDonagh reported a case study of 23-year-old female with a persistent developmental stutter since age five. Fluidity of speech was assessed before and after instruction. The \% of dysfluencies dropped from $6.8 \%$ per minute before intervention to $0.8 \%$ after intervention. The rate of speech measured in syllables per minute increased from 175.2 before intervention to 259.2 after intervention. The intervention required looking down in the direction of her navel at the start of any word on which she predicted difficulty. This success was replicated with a 5-yearold girl, who could resolve her stuttering behaviour by saying the words she was stuck on to her tummy. This viability approach is to be piloted in RCT. If modifying our tongue position can have such a powerful effect on neurological functioning in DS, a future direction in research must be to ascertain what learned movement patterns alter tongue position and how they could be modified.

\section{Acknowledgement}

The research is being sponsored by the Irish Research Council.

\section{References}

1. BBC Radio (2019) Stephen Fry: What Makes Us Human. [podcast] What Makes Us Human with Jeremy Vine.

2. Bart de Boer (2016) Evolution of speech and evolution of language. Psychonomic Bulletin \& Review, 24(1): 158-162.

3. Isha Foundation (2016) Aum, Amen, Ameen - One And The Same.

4. Tversky B (2019) Mind in Motion: How Action Shapes Thought. Basic Books.

5. Boysson Bardies B (2001) How language comes to children. Cambridge Mass, MIT Press.

6. Guitar B (2019) Stuttering: An Integrated Approach to its Nature and Treatment. NEW YORK: Wolters Kluwer Medical.

7. Boyle M, Blood GW, Blood IM (2009) Effects of perceived causality on perceptions of persons who stutter. J Fluency Disord 34(3): 201-218.

8. Craig A, Blumgart E, Tran Y (2009) The impact of stuttering on the quality of life in adults who stutter. J Fluency Disord 34(2): 61-71.

9. Yairi E, Ambrose N (2013) Epidemiology of stuttering: 21st century advances. J Fluency Disord 38(2): 66-87.

10. Christian Büchel, Martin Sommer (2004) What Causes Stuttering? Plos Biology 2(2): e46.
11. Briley P (2018) Issues in Assessment of Children Who Stutter: A Survey of Speech-Language Pathologists in the State of North Carolina. Clinical Archives of Communication Disorders 3(2): 95-103.

12. Nye C, Vanryckeghem M, Schwartz JB, Herder C, Turner HM (2013) Behavioral Stuttering Interventions for Children and Adolescents: a Systematic Review and Meta-Analysis. J Speech Lang Hear Res 56(3): 921-932.

13. Effective Stuttering Treatment Project (EST) - Department of Special Needs Education (2019).

14. McDonagh H (2018) Mechanics of the Frontal Aslant Tract. Journal of Neurology \& Neurophysiology 9: 11.

15. Catani M, Dell'acqua F, Vergani F, Malik F, Hodge H, et al. (2012) Short frontal lobe connections of the human brain. Cortex 48(2): 273-291.

16. Dick AS, Garic D, Graziano P, Tremblay P (2019) The frontal aslant tract (FAT) and its role in speech, language and executive function. Cortex 111: $148-163$

17. Sanders I, Mu L (2013) A Three-Dimensional Atlas of human Tongue Muscles. Anat Rec (Hoboken) 296(7): 1102-1114.

18. Bahr D (2011) Nobody Ever Told Me (or my Mother) That!: Everything from Bottles and Breathing to Healthy Speech Development. Cork: BookBaby.

19. McDonnell P, Corkum, V (1991) The role of reflexes in the patterning of limb movements in the first six months of life. Advances in Psychology 81: 151-173.

20. Etchell A, Adhikari A, Weinberg LS, Choo AL, Garnett EO, et al. (2018) A systematic literature review of sex differences in childhood language and brain development. Neuropsychologia 114: 19-31.

21. Patton MH, Blundon JA, Zakharenko SS (2019) Rejuvenation of plasticity in the brain: opening the critical period. Curr Opin Neurobiol 54: 83-89.

22. Farrant K, Uddin LQ (2015) Asymmetric development of dorsal and ventral attention networks in the human brain. Developmental Cognitive Neuroscience 12: 165-174.

23. Cooper E, Mackey A (2016) Sensory and cognitive plasticity: implications for academic interventions. Current Opinion in Behavioral Sciences 10: 21-27.

24. Garic D, Broce I, Graziano P, Mattfeld A, Dick AS (2018) Laterality of the frontal aslant tract (FAT) explains externalizing behaviors through its association with executive function. Developmental Science 22(2): e12744.

25. Diaz G, Cooper J, Rothkopf C, Hayhoe M (2012) Internal Models for Predictive Saccades in a Natural Interception Task. Journal of Vision 12(9): 606-606.

26. Smythies J (2005) How the brain decides what we see. J R Soc Med 98(1): 18-20.

27. Marshall AC, Gentsch A, Schütz Bosbach S (2018) The Interaction between Interoceptive and Action States within a Framework of Predictive Coding. Front Psychol 9: 180.

28. Richardson RT, DeLong MR (1990) Context-dependent responses of primate nucleus basalis neurons in a go/no-go task. J Neurosci 10(8): 2528-2540.

29. Aston Jones J, Rajkowski J, Cohen J (1999) Role of locus coeruleus in attention and behavioral flexibility. Biol Psychiatry 46(9): 1309-1320.

30. Lisman J, Grace AA, Duzel E (2011) A neoHebbian framework for episodic memory; role of dopamine-dependent late LTP. Trends Neursci 34(10): 536-547.

31. Gremmler S, Lappe M (2016) Saccadic Adaptation Is Associated with Starting Eye Position. Front Hum Neurosci 10: 322. 
32. Alahyane N, Pélisson D (2005) Long-lasting modifications of saccadic eye movements following adaptation induced in the double-step target paradigm. Learn Mem 12(4): 433-443.

33. Jackson ES, Gerlach H, Rodgers NH, Zebrowski PM (2018) My Client Knows That He's About to Stutter: How Can We Address Stuttering Anticipation during Therapy with Young People Who Stutter? Semin Speech Lang 39(4): 356-370.

34. Brutten GJ, Janssen P (1979) An Eye-Marking Investigation of Anticipated and Observed Stuttering. J Speech Hear Res 22(1): 20-28.

35. Higuchi S, Chaminade T, Imamizu H Kawato M (2009) Shared neural correlates for language and tool use in Broca's area. NeuroReport 20(15): 1376-1381.

36. Parker A, Parkin A, Dagnall N (2013) Effects of Saccadic Bilateral Eye Movements on Episodic and Semantic Autobiographical Memory Fluency. Front Hum Neurosci 7: 630.
37. Shapiro F (2014) The Role of Eye Movement Desensitization and Reprocessing (EMDR) Therapy in Medicine: Addressing the Psychological and Physical Symptoms Stemming from Adverse Life Experience. Perm J 18(1): 71-77.

38. Yamaguchi H, Sueishi K (2003) Maloccusion Associated with Abnormal Posture. Bull Tokyo Dent Coll 44(2): 43-54.

39. Schmidt JE, Carlson CR, Usery AR, Quevedo AS (2009) Effects of tongue position on mandibular muscle activity and heart rate function. Oral Surg Oral Med Oral Pathol Oral Radiol Endod 108(6): 881-888.

40. Di Vico R, Ardigò LP, Salernitano G, Chamari K, Padulo J (2019) The acute effect of the tongue position in the mouth on knee isokinetic test performance: a highly surprising pilot study. Muscle Ligaments and Tendons Journal 3(4): 318-323.

41. Little AC, Jones BC, DeBruine LM (2011) Facial attractiveness: evolutionary based research. Philos Trans R Soc Lond B Biol Sci 366(1571): 1638-1659 